\title{
Kinematics of Stars in Kapteyn Selected Area 71: Sampling the Monoceros and Sagittarius Tidal Streams
}

\author{
Dana I. Casetti-Dinescu ${ }^{1,2,3}$, Jeffrey L. Carlin ${ }^{4}$, Terrence M. Girard ${ }^{2}$, Steven R. Majewski ${ }^{4}$, \\ Jorge Peñarrubia ${ }^{5}$ and Richard J. Patterson ${ }^{4}$
}

\begin{abstract}
We present a 3D kinematical analysis of stars located in Kapteyn Selected Area $71\left(l=167.1^{\circ}, b=-34.7^{\circ}\right)$, where previously a stellar excess was found (Dinescu et al. 2002, Newberg et al. 2002). Previous findings indicated that the stellar excess has a cold kinematical signature as inferred from proper motions, and was initially associated with debris from the Sagittarius dwarf galaxy (Sgr) - namely the southern trailing tail. We have obtained radial velocities using the Hydra multiobject spectrograph on the WIYN 3.5 m telescope. Results for 183 proper-motion selected stars indicate that the dominant population in this stellar excess is not debris from Sgr, but rather a population that kinematically belongs to the ring-like stream that is now known as the Monoceros stream. The orbit determined for this population agrees very well with the predictions for the Monoceros stream from Peñarrubia et al. (2005). The radial-velocity dispersion of this population is between 20 and $30 \mathrm{~km} / \mathrm{s}$, lower than that of the Galactic field. Also, the shape of the radial-velocity distribution shows a sharp cut-off on one side, which is more in line with model predictions of the disruption of a satellite rather than with the distribution of the Galactic field. Despite the fact that we now believe most of the stars in the stellar excess to be part of Monoceros, about ten stars in this stellar excess have highly negative radial velocities, which is a clear indication of their membership to the Sgr trailing tidal tail.
\end{abstract}

Subject headings: Galaxy:structure - Galaxy: kinematics and dynamics

\footnotetext{
${ }^{1}$ Astronomy Department, Van Vleck Observatory, Wesleyan University, Middletown, CT 06459

${ }^{2}$ Astronomy Department, Yale University, P.O. Box 208101, New Haven, CT 06520-8101

${ }^{3}$ Astronomical Institute of the Romanian Academy, Str. Cutitul de Argint 5, RO-75212, Bucharest 28, Romania

${ }^{4}$ Department of Astronomy, University of Virginia, P.O Box 400325, Charlottesville, VA 22904-4325

${ }^{5}$ Department of Astronomy, University of Victoria, Victoria, BC, Canada
} 


\section{Introduction}

The wealth of streams and overdensities discovered recently in the Milky Way halo (e.g., Belokurov et al. 2006, Grillmair 2006, Vivas and Zinn 2006) while spectacular, has made our task of understanding the halo more challenging than some ten years ago. A thorough knowledge of these structures' kinematics is key to understanding their origin and significance as building blocks of the Galaxy in the hierarchical view of its formation.

In this contribution, we focus on a region in the sky where two major tidal streams appear to reside at different distances: the Sagittarius (Sgr) tidal stream, and the Monoceros stream/structure (hereafter Mon). While the Sgr tidal stream is better understood, Mon has a more ambiguous origin because its progenitor has not yet been established. Mon was "pieced together" by the discovery of stellar overdensities roughly parallel to the Galactic plane, and above and below the plane. Many large-area photometric surveys have contributed to the mapping of Mon: starting with SDSS (Newberg et al 2002, Yanny et al. 2003) and continuing with 2MASS (Rocha-Pinto et al. 2003), the INT/WFC (Ibata et al 2003, Conn et al. 2005) and the AAT/WFI (Conn et al. 2007). It was realized by Rocha-Pinto et al. (2003) and Ibata et al. (2003), that this structure is immense, spanning from $l=100^{\circ}$ to $270^{\circ}$, and that it is located in a ring-like form at a Galactocentric distance of $\sim 18 \mathrm{kpc}$.

Our region of interest is a $40^{\prime} \times 40^{\prime}$ area centered on Kapteyn Selected Area (SA) 71, at $(l, b)=\left(167.1^{\circ},-34.7^{\circ}\right)$. The location of SA 71 is within the general area of the Monoceros stream detections (see e.g., Fig. 3 in Rocha-Pinto et al. 2003). Our previous study of this region (Dinescu et al. 2002, hereafter D02) included the determination and analysis of absolute proper motions and photographic photometry of stars within $17<V<20$. D02 have demonstrated that SA 71 contains an excess of blue stars when compared to a Galactic model that was well fit in three other lines of sight. The proper motions of the excess stars show a tight clump, also supporting the notion of a kinematically cold substructure. At that time, the only well-known tidal stream was that of Sgr, and D02 have interpreted their data as the Sgr stream. Specifically, the trailing tidal tail that arches underneath the Sun's location to reach the Galactic plane toward the Anticenter direction (e.g., Majewski et al. 2003) best explained the data in D02: Galactic location, colors and magnitudes of excess stars, and approximately the mean absolute proper motion. Here, we present radial velocities of stars selected in the excess region of the color-magnitude diagram (CMD) and in the proper-motion diagram (also known as the vector-point diagram, VPD), and show that, while a small number of stars in this excess belong to Sgr, most of them are part of a different structure. The kinematical properties of this structure are now best explained in the general context of the Monoceros structure.

The current study is part of a larger proper-motion and radial-velocity survey in over 50 
SAs. The characteristics of this survey, as well as the astrometric techniques are throughly described in Casetti-Dinescu et al. (2006, CD06). We will therefore refer the interested reader to that contribution for more specific aspects of the reductions. All proper motion units throughout the paper are mas/yr, and radial velocities are heliocentric, unless otherwise specified.

The paper is structured as follows. In Section 2 we present our data: photometry, proper motions and radial velocities, in Section 3 we discuss our results and compare the observed kinematics with the predictions of the Besançon Galactic model (Robin et al. 2003). In Section 4 we compare our results with the predictions of the Peñarrubia et al. (2005, hereafter P05) model of the Mon as a disrupting satellite. In Section 5 we discuss the possibility that the Galactic warp and/or other nearby overdensities may be present in SA 71. Section 6 outlines our conclusions.

\section{The Data}

\subsection{Photometry and Proper Motions}

The set of 14 photographic plates used in this study includes 11 Mayall $4 \mathrm{~m}$ plates (epoch=1975 and 1996), two Du Pont 2.5 m plates (epoch=1996), and one Mt. Wilson 60" plate (epoch=1912). The Du Pont and Mt. Wilson plates contain two exposures each: a 3 minute and a 60 minute. All plates were scanned with the Yale PDS microdensitometer, based on an input list of objects. This list was determined by combining coarser scans of six Mayall plates, which are the deepest in our set. Both the photographic photometry and the proper-motion determinations were described in D02 and CD06, and therefore we refer the reader to these papers for detailed measurements and reduction procedures. The photographic photometry was calibrated to standard Johnson $B V$ magnitudes using CCD photometry obtained with the Swope $1 \mathrm{~m}$ telescope at Las Campanas in 2001 (see D02). The CCD photometry covered $23 \%$ of the total area covered by the photographic plates and to a limiting magnitude $B=22$, while the Mayall $4 \mathrm{~m}$ photographic plates are deeper by 0.7 mags in $B$. From the comparison of photographic and CCD magnitudes, and assuming that the CCD magnitude errors are substantially smaller than the photographic ones, we have

estimated that the photographic $V$ uncertainties are 0.05 mags for $V=17$ to 21 , and the $B-V$ uncertainties are 0.08 mags, for the same $V$ range. Outside this $V$-magnitude range, the uncertainties increase rapidly due to saturation of the photographic images at the bright end, and due to the decrease in $S / N$ at the faint end.

In this work, we have relied on the SExtractor resolution classifier (Bertin \& Arnouts 
1996) for object classification, using the deepest, best-seeing, Mayall $4 \mathrm{~m}$, modern-epoch plate (\#4323, IIIa-F + GG495). The value of the stellarity index that separates galaxies from stars was determined from a number of criteria: 1) visual inspection of images, 2) plots of image parameters such as image radius and peak density, and 3) comparison with the 2MASS classifier for objects with $V<19$. For another SA field - namely SA 94 - that has similar plate material to SA 71 (see CD06), together with SDSS photometry and object classification, we can also compare our object classification with that of SDSS. We obtain that our classification of galaxies recovers $75 \%$ of the SDSS galaxies at $V=21.0$ and $50 \%$ at $V=21.6$.

The astrometric reductions have been redone in this work - compared to that of D02 to include the measurements of the old Kapteyn, Mt. Wilson plate. This expands the time baseline from $\sim 20$ to 84 years, but only for stars brighter than $V \sim 18$. Also, we have used a list of well-measured, round-shaped galaxies to model magnitude-dependent systematics in the proper motions. The reduction procedure has been described in D02 and in Sections 2.5 and 2.6 of CD06, and will not be repeated here. The galaxies' proper motions (or positional residuals) do not show significant trends as a function of color, for $0.3<B-V<1.8$, or magnitude, in the range $V=18$ to 21.5. The correction to absolute proper motion is determined by galaxies, selected using the following criteria: $V=18$ to 21.5 , measured on at least six plates, and with proper-motion size less than 30 mas/yr. This sample includes 618 galaxies. We have then used probability plots (Hamaker 1978) trimmed at $10 \%$ on this sample to estimate its mean and dispersion. We obtain an average relative proper motion for the galaxy sample of $\left(\mu_{\alpha} \cos \delta, \mu_{\delta}\right)=(-2.32 \pm 0.18,2.28 \pm 0.19)$ mas/yr, which is applied as an offset to all objects; this number is also known as the correction to absolute proper motion.

For stars, we obtain formal proper-motion uncertainties of 0.5 mas/yr for $V=18,0.9$ mas $/ y r$ for $V=19,1.4 \mathrm{mas} / \mathrm{yr}$ for $V=20$, and $2.2 \mathrm{mas} / \mathrm{yr}$ for $V=21$, in each coordinate.

\subsection{Radial Velocities}

\subsubsection{Sample Selection}

The stars selected for followup spectroscopy are within the magnitude and color ranges where the stellar excess was found in D02. Specifically, the approximate color range is $0<(B-V)<1.1$ and magnitude range is $18<V<19.5$. Likewise, stars were selected within $\mathrm{a} \sim 3 \mathrm{mas} / \mathrm{yr}$ radius from the proper-motion center of the tight clump defined by the excess, blue stars. A few stars outside these ranges were also considered, for comparison 
purposes. The program stars for spectroscopy are shown in Figure 1 as bold symbols among the general stellar population of the field; selections in the color-magnitude diagram (CMD) and the vector-point diagram (VPD) are shown.
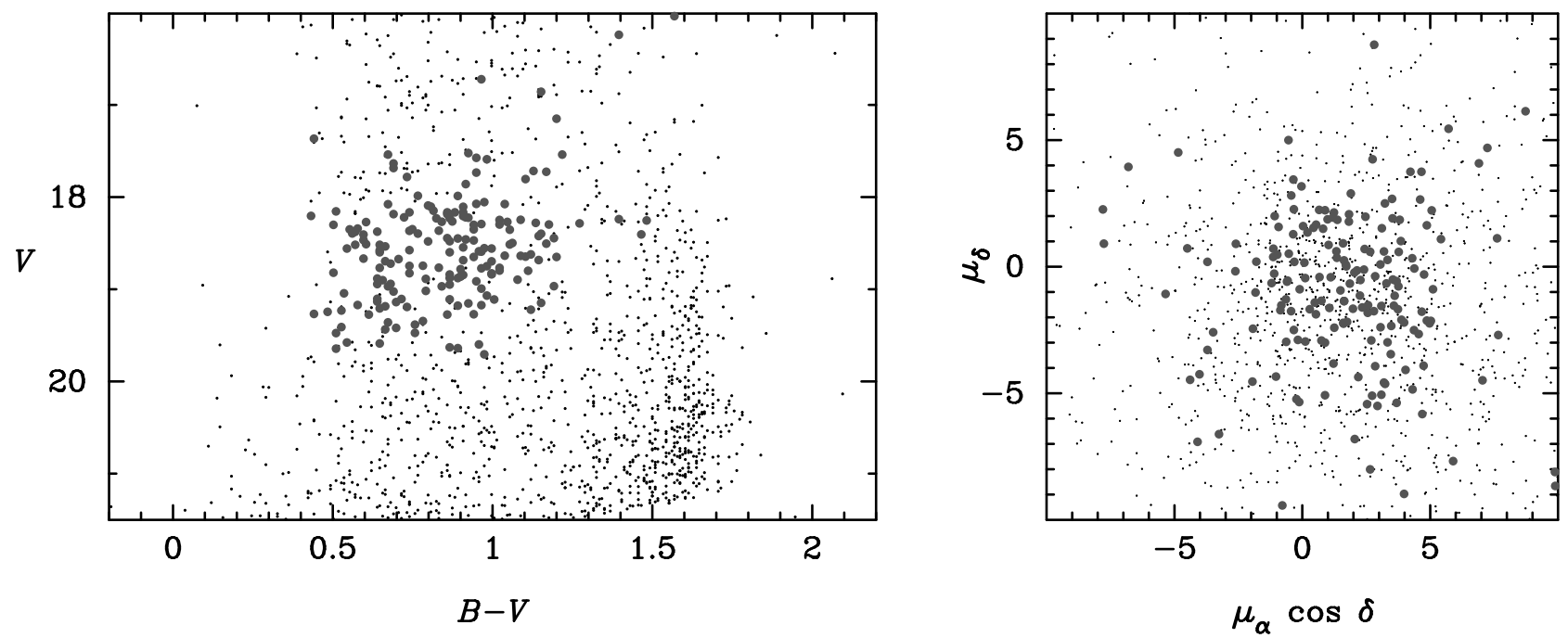

Fig. 1. - The selection of stars (filled circles) for RV measurements in the CMD (left) and in the VPD (right).

\subsubsection{Observations}

Radial velocities (RVs) were obtained from observations taken with the $3.5 \mathrm{~m}$ WIYN telescope during six observing runs. We have used the Hydra multifiber positioner with the WIYN bench spectrograph in two setups. The first one (Dec. 2002, Nov. 2003 observing runs) used the 800@30.9 grating with the red fiber cables and an order centered in the neighborhood of the $\mathrm{Mg} b$ triplet $(5170 \AA)$, and covering about $980 \AA$ of the spectrum. This corresponds to a dispersion of $0.478 \AA \mathrm{pix}^{-1}$, and a spectral resolution of $0.956 \AA$. The comparison source was a $\mathrm{Cu}$-Ar lamp. The second spectrograph configuration (Dec. 2005, Oct. 2006, Dec. 2006 and Dec. 2007) used the 600@10.1 grating with the red fiber cable to yield a wavelength coverage $\lambda=4400-7200 \AA$ at a dispersion of $1.397 \AA$ pix $^{-1}$, for a spectral resolution of $3.35 \AA$. Each of these 2005-7 datasets was obtained in less than optimal conditions, including substantial scattered moonlight in Dec. 2005, and cloudy conditions in the 2006 and 2007 runs.

Table 1 contains the summary of the observations as follows: the first column lists the dates of the observing runs, the second column the number of Hydra configurations observed during the respective run, the third column the total exposure time, the fourth column the 
central wavelength, the fifth column the dispersion. The sixth column indicates the number of RV standard stars observed, and the total number of individual standard star spectra taken. The seventh column lists the number of stars with a Tonry-Davis ratio (TDR, Tonry \& Davis 1979) larger or equal to 8, and the last column shows the $V$-magnitude range of the program stars observed in each run. Each Hydra configuration was exposed in sets of 30 min, to obtain the total exposure time listed. For RV calibrations, each night we observed standard stars which were typically RV-calibrated HD stars listed in the Astronomical Almanac as well as stars in M 67 (Mathieu et al. 1986) selected to be single, well-measured stars. On the 2005-6 observing runs, each individual HD standard was observed through multiple Hydra fibers, to yield multiple, distinct cross-correlation template spectra (with that total number also listed in Table 1). The magnitude range of the standard stars is $V=7.3$ to 11.6, therefore these are well-measured stars with high $\mathrm{S} / \mathrm{N}$.

Table 1: Summary of Observations

\begin{tabular}{cccccccc}
\hline Date & Config. & $\begin{array}{c}\text { Exp. } \\
(\text { hours })\end{array}$ & $\begin{array}{c}\text { Wavelength } \\
(\AA)\end{array}$ & $\begin{array}{c}\text { Dispersion } \\
\left(\AA \text { pix }^{-1}\right)\end{array}$ & $\begin{array}{c}\text { RV standards } \\
\text { \# of distinct spectra })\end{array}$ & N $_{T D R \geq 8}$ & $V$-range \\
\hline Dec 2002 & 1 & 2 & 5170 & 0.478 & 2 HD (2) & 37 & $18-19$ \\
Nov 2003 & 2 & 5,2 & 5170 & 0.478 & 2 HD+15 M67 (17) & 74 & $18-19$ \\
Dec 2005 & 1 & 3 & 5800 & 1.397 & 1 HD (5) & 27 & $18.5-19.5$ \\
Oct 2006 & 1 & 3 & 5800 & 1.397 & 11 HD (65) & 47 & $18.2-19$ \\
Dec 2006 & 1 & 2 & 5800 & 1.397 & 2 HD (10) & 58 & $16.7-19.3$ \\
Dec 2007 & 1 & 4 & 5800 & 1.397 & 9 HD (40) & 55 & $16.0-19.7$ \\
\hline
\end{tabular}

The data were reduced using the DOHYDRA reduction package in IRAF after the standard pre-processing was applied to the initial two-dimensional spectra. For the wavelength calibration we have used 15-17 comparison lines. The sky subtraction was done outside the DOHYDRA package, for each observation individually, with typically 10 fibers to define it. Then, for each Hydra configuration, the multiple sets of 30-minute observations were median combined. Finally, RVs were derived using the FXCOR task in IRAF to cross-correlate these target spectra against all RV standard spectra.

Comparing the RVs obtained from our observations of the standard stars during the 2003 run with those from the literature, we obtain that the RV difference is $0.03 \pm 0.26 \mathrm{~km} / \mathrm{s}$, and the standard deviation is $1.1 \mathrm{~km} / \mathrm{s}$. As mentioned above, these are bright ( $V=7.3$ to 11.6$)$, well-measured stars. Our program stars are however much fainter (see Tab. 1). Therefore, we have kept only those determinations with a $\mathrm{TDR} \geq 8(S / N \geq 15)$. 

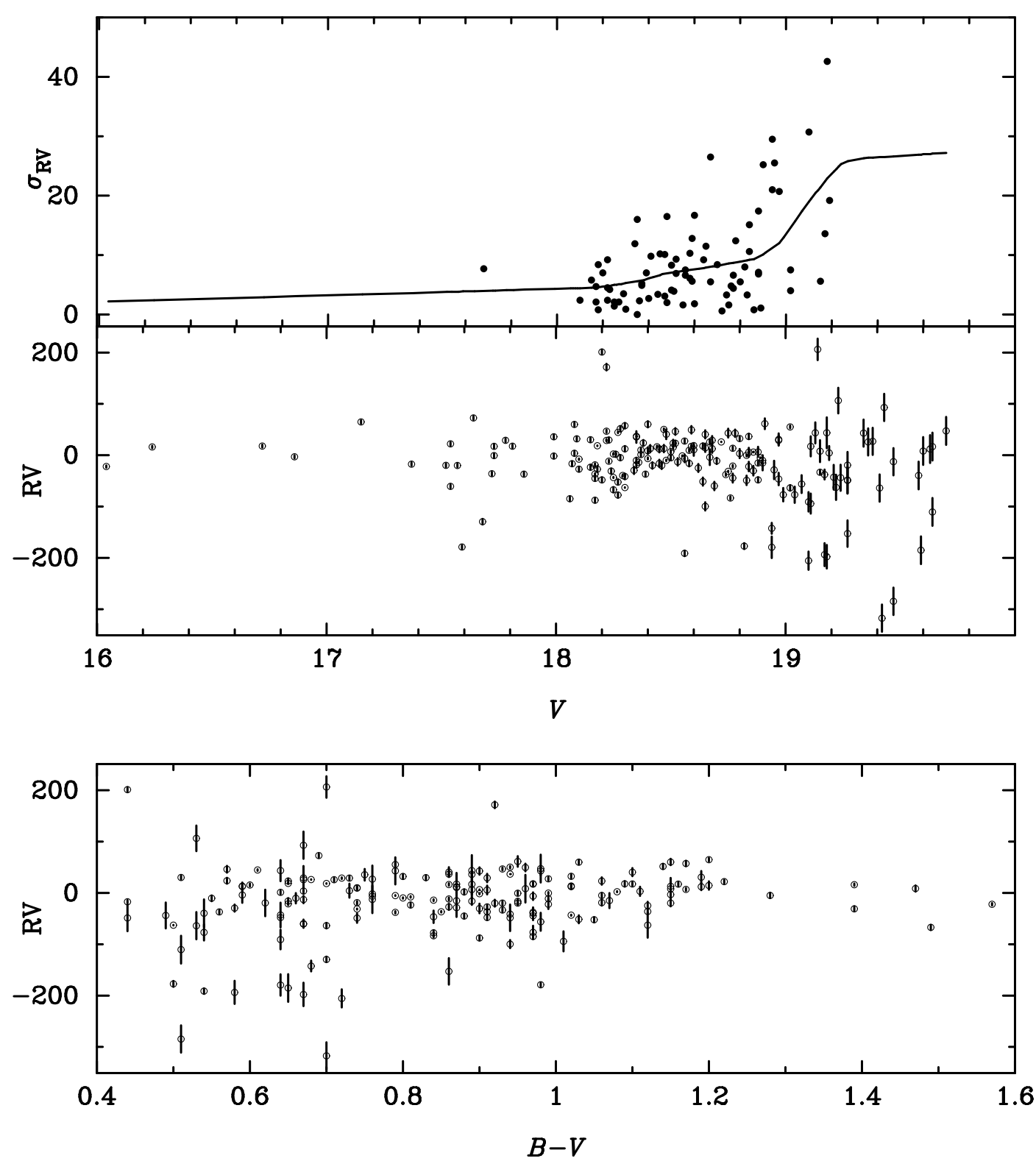

Fig. 2.- The RV scatter for stars with multiple measurements as a function of magnitude (top). The final RVs as a function of magnitude (middle) and as a function of color (bottom) for all of the stars in our sample. Units are $\mathrm{km} / \mathrm{s}$.

The Hydra configurations were designed to have some overlap between different sets of observations. Comparing results from different runs, where the overlap includes between 7 and 20 stars, we obtain the following. The RV errors are between 5 and $10 \mathrm{~km} / \mathrm{s}$ per star, per single measurement for $V=18$ to 18.8 . For fainter stars, the errors increase rapidly to reach $\sim 20 \mathrm{~km} / \mathrm{s}$ at $V \sim 19.0$. We have also found significant (2 to $5 \sigma$ ) RV offsets between 
different runs; these are of the size of 5 to $10 \mathrm{~km} / \mathrm{s}$. Our two best RV-standard calibrated runs, the 2003, 5-h integration set, and the Oct. 2006 set, have an offset of $-1.2 \pm 3.2 \mathrm{~km} / \mathrm{s}$ for eight stars in common between the two runs. To place all observations on the same system, we have chosen the 2003, 5-h integration run as our "reference" system, and we have offset the other sets onto this system. For stars with multiple RV determinations, we take the average as the final RV value, and the RV error is calculated from the scatter of the determinations. For stars with a single RV determination, we adopt RV errors as follows: First, from stars with multiple determinations, we plot the RV scatter as a function of $V$ magnitude. We then apply a moving median (0.5 mag bin) to derive a curve that describes the dependence of RV error as a function of magnitude. Finally, for each star of a given magnitude, we interpolate the derived dependence of RV error with magnitude to determine the RV error. In Figure 2 (top), we show the RV scatter as a function of magnitude for stars with multiple determinations, and the adopted curve for stars with single determinations. The middle plot shows the RVs with their error bars as a function of magnitude, while the bottom plot shows the RVs as a function of $B-V$ colors. Our entire sample contains 183 stars.

\section{Discussion of the Observations}

We proceed now to interpret our results in light of the three most plausible contributors to the stellar counts in this region of the sky: the Galactic field, the Sgr tidal stream and the Monoceros stream.

To compare our data with expectations for the stellar Galactic field, we have used the simulated catalogs obtained with the Besançon Galactic model (Robin et al. 2003). The simulated data were generated in a 0.43 degree square field for stars between $V=14$ to 22 . The photometry of the model was convolved with a $0.05 \mathrm{~V}$-mag error and a 0.08 mag color error in $(B-V)$, which is a good representation of our uncertainties between $V=17$ to 21 , where the comparison is made (see Section 2.1). The proper-motion errors to be convolved with the model are derived from the run of observed proper-motion errors as a function of magnitude (Section 2.1). After eliminating all stars with formal errors larger than 4 mas/yr, we fit a second-order polynomial to the observed dependence of proper-motion errors with magnitude in the range $V=14$ to 22 . The coefficients thus obtained are input into the Besançon model. Similarly, for radial velocities, we determine the quadratic dependence of

formal errors as a function of magnitude as given by the data in the range $V=17.5$ to 19 (Section 2.2) and input this function into the Besançon model.

For the Sgr stream, we use the disruption model within a spherical potential from Law 
et al. (2005). In this contribution, we do not fully analyze the kinematical data for Sgr, but rather use the Law et al. model as a guideline for identifying the Sgr population and its likely kinematics. A future contribution will be entirely dedicated to the kinematical analysis of the Sgr southern trailing tail, and it will include more SA fields besides SA 71.

For the Mon disruption model we use the predictions from P05, and dedicate Section 4 to this kinematical comparison.

\subsection{What is Creating the SA 71 Starcount Excess ?}

In our D02 paper we have compared $(B-V)$ color distributions in three $V$-magnitude bins with those predicted by another Galactic model (Méndez \& van Altena 1996) in four SA fields, including SA 71. We have shown there that, while three of the SA fields have color distributions that agree with the Galactic model, SA 71 has excess counts at blue colors, $(B-V)<1.1$. Specifically, the excess counts were found to peak at $(B-V)=1.0$ for $V=18-19$, and at $(B-V)=0.6$ for $V=19-20$ (see Fig. 1 in D02). In the bright magnitude bin, the observations indicated 219 stars, and the model predicted 167, while in the faint magnitude bin, the observations found 256 stars, while the model predicted 210.

In this contribution, we will not repeat the starcount analysis from D02, as we are mainly interested in the kinematical aspect. Instead, we show in Figure 3 the CMDs as given by the Besançon model (top) and by our data (middle) for a qualitative comparison. We have applied a $V=22.0$ magnitude limit in our data, since the quality is rather poor beyond this value. For the model, which was initially generated within $V=14$ to 22 , we have applied a $B=22.9$ limit, to mimic the blue photographic plate limit of the data. From Figure 3, the excess of stars in the observations compared to the model is readily visible at $(B-V)<1.1$ and $V \geq 18$.

To better illustrate the stellar excess present at blue colors in the observations we show $(B-V)$ color distributions for four $V$-magnitude bins in Figure 4 . The observations are represented with a continuous black line, while the model predictions are shown with a grey line. The magnitude range is specified in each panel. It appears that the Besançon model overpredicts starcounts for stars redder than $B-V=1.3$ in all magnitude ranges; these stars are red dwarfs belonging to the thin disk population. While our purpose here is not to constrain the model with our observations, an adjustment of the thin-disk parameters can account for this discrepancy. The stellar excess in the observations when compared to the model predictions is obvious for colors bluer than $(B-V)<1.2$, in all three of the faintest magnitude bins. 
When the D02 paper was written, the most well-known disrupting satellite was Sgr. Its orbit projected onto the sky indicated that Sgr debris can be found in SA 71 (D02), more specifically, debris from the southern trailing tail (e.g., Majewski et al. 2003). Additionally, D02 noted that three carbon stars from the catalog of Totten and Irwin (1998) that are near SA 71 , have highly negative RVs $(\sim-140 \mathrm{~km} / \mathrm{s})$ that match the values for Sgr tidal debris. Later, Putman et al. (2004) found an H I stream along the orbit of Sgr, toward the Galactic anticenter and encompassing SA 71. The highly negative line-of-sight velocity range (-380 to $-180 \mathrm{~km} / \mathrm{s}$, Putman et al. 2004) of the H I stream is indicative of a likely membership to the Sgr trailing trail according to the stellar Sgr velocities by Majewski et al. (2004). Putman et al. (2004) interpreted this H I stream as a gaseous component of Sgr stripped by ram pressure, as the satellite passed through the edge of the Galactic disk. In this context, D02 interpreted the stellar excess at $(B-V) \sim 1.0, V=18-19$, as stellar debris from Sgr, specifically red-clump/horizontal-branch stars at $d_{\odot} \sim 30 \mathrm{kpc}$. The proper motions too were approximately compatible with those predicted for Sgr tidal debris (D02). In the meantime, photometric surveys revealed another very extended structure: the Monoceros ring. Mon is now best understood as a tidal feature from a disrupted satellite with a low-inclination, circular orbit (e.g., P05, Conn et al. 2007 and references therein), although its originating satellite is still a matter of debate (Conn et al. 2007 and references therein). SA 71 lies in the general area where Mon debris was mapped below the Galactic plane (CD06 and references therein), and where the P05 model predicts tidal debris. The P05 model predicts Mon debris at a heliocentric distance $d_{\odot} \sim 9-10 \mathrm{kpc}$, in the region of SA 71 .

Therefore, assuming that in SA 71 we have tidal debris from both Sgr and Mon, we have used the Girardi et al. (2002) isochrones to qualitatively compare the stellar excess seen in SA 71 to the likely populations of Sgr and Mon. We have used the 10 Gyr-old isochrones of $[\mathrm{Fe} / \mathrm{H}]=-0.7,-1.3$ and -1.7 , which are a reasonable approximation for the stellar populations in discussion. The two most metal rich isochrones were placed at a distance of $9 \mathrm{kpc}$, and they were chosen to approximately represent populations in Mon (Rocha-Pinto et al. 2003, Ibata et al. 2003, Yanny et al. 2003). The most metal poor isochrone was placed at a distance of $39 \mathrm{kpc}$, and was chosen to represent Sgr (Newberg et al. 2002), although more metal rich stars in Sgr's trailing tail are also present (Majewski et al. 2003). The isochrones were shifted to account for the reddening of $E_{B-V}=0.16$ at the center of our SA 71 field (Schlegel, Finkbeiner \& Davis 1998). The bottom plot of Figure 3 shows our data together with the isochrones. The dark, filled symbols represent Sgr candidate members according to their RVs (see Section 3.2). The subgiant, turnoff and possibly main sequence features of the isochrones at $9 \mathrm{kpc}$ best explain the stellar excess which is redder at $V=18-19$ and bluer at $V=19-20$. Also, the $V$-mag location of the red clump for the isochrone at $39 \mathrm{kpc}$ lies in the subgiant/turnoff region of the isochrones representing Mon. As already noted, 
a more metal rich population in Sgr, which is in agreement with the presence of M giants in the trailing tail (Majewski et al. 2003), would shift the red clump toward redder $B-V$ colors right in the region were the bright, redder stellar excess was found thus justifying the D02 interpretation of the excess.

At fainter magnitudes $(V \geq 21)$, there appears to be another prominent stellar excess at $(B-V)=0.3-0.7$, which can be best interpreted as turnoff stars in Sgr. While photometric uncertainties are large here (both precision and calibration), and the stellar/galaxy discrimination is poorer (see Section 2.1), we believe that most objects are stars at these blue colors. We hope to be able to re-address this issue with deep CCD photometry in a future contribution.

\subsection{Kinematical Clues to the Stellar Populations and Streams in SA 71}

\subsubsection{Radial Velocities}

In Figure 5, we show the distribution of RVs for the Besançon model and for our data, as a function of color (left panels) and magnitude (middle panels). Normalized histograms of the RV distributions are also plotted in the middle panels of Fig. 5, to show the shape of the distributions. Along with the RV distributions, we show the VPD (right panels) for each sample. The Besançon data were trimmed in color $(0.4<B-V<1.2)$ and magnitude $(18.0<V<19.7$ ), to match the selection of our RV sample (Fig. 1, and eliminating stars brighter than $V=18$ ). No other selection was applied to the Besançon data, while the observed RV sample was selected in proper-motion space to preferentially pick out members in the "cold" VPD structure. Within the CMD selection $0.4<B-V<1.2$ and $18.0<V<19.7$ our observations contain 229 stars of which 158 have RVs (see also Fig. 1). For the same CMD selection, the Besançon model has 130 stars.

The first row of panels in Fig. 5 shows the Besançon distributions, where errors are convolved into the model, but not represented with error bars, for clarity. The bottom three rows of panels show our observed distributions separated into three groups: all stars with RVs (second row), stars with RVs and proper motions outside the clump in the VPD (third row), and those stars with proper motions inside the VPD clump (fourth row). The proper-motion clump is selected within a $\sim 2 \sigma$ radius of the proper-motion centroid.

The first thing that stands out in Figure 5, is the group of stars at RV $\sim-180 \mathrm{~km} / \mathrm{s}$, which also have blue colors and faint magnitudes. At the location of SA 71, the predicted heliocentric radial velocity for Sgr tidal debris is $\sim-170 \mathrm{~km} / \mathrm{s}$ (e.g., Law et al. 2005 models, in a spherical potential). Majewski et al. (2004, their Fig. 2) find similar RV values for 
M giants in a similar part of the Sgr trailing tail. It is this velocity component that best separates Sgr members from the Galactic field population. In the Besançon model, there are two such stars, while in our data there are nine. The tenth Sgr RV candidate is at $V=17.6$ (Fig. 3). If we choose only stars within the tight proper-motion clump, there are six stars with highly negative RVs that match the velocity predicted for Sgr debris. Therefore, in the magnitude range explored here, a small number of stars in our tight proper-motion clump most likely belong to Sgr.

The majority of the stars in the proper-motion clump, however, are not Sgr members, according to the RVs. Moreover, they show a smaller RV dispersion than that of the Galactic field (rows 1 and 4 in Fig. 5) between $V=18$ and 19. For magnitudes fainter than $V=19$, uncertainties in RVs increase substantially (see Fig. 2), and thus we can not characterize well the intrinsic RV dispersion at these magnitudes. Also, the shape of the RV distribution of the observed data has a sharp cutoff at $\mathrm{RV} \sim 60 \mathrm{~km} / \mathrm{s}$, while that of the Besançon model has a gradual decline. The observed RV distribution of stars with proper motions outside the VPD clump (Fig. 5, row 3) is more in line with the RV distribution of the Besançon model. The RV dispersion of the excess stars within the proper-motion clump is rather difficult to determine accurately, given the overlap with Galactic field stars in both RV and propermotion space. Nevertheless, we can estimate it giving upper and lower limits as follows. First, from the stars within the proper-motion clump (Fig. 5, bottom row), we eliminate all stars fainter than $V=19$, where RV errors increase substantially (Fig. 2), and with RV size larger than $100 \mathrm{~km} / \mathrm{s}$. The average for the 81 stars thus selected, is $-0.9 \pm 3.7 \mathrm{~km} / \mathrm{s}$, with $\sigma_{R V}=33 \mathrm{~km} / \mathrm{s}$. If we consider only stars within $V=18.3$ to 18.6 , where the smallest scatter is apparent, then we obtain an average $9.9 \pm 4.5 \mathrm{~km} / \mathrm{s}$ and a dispersion $\sigma_{R V}=23$ $\mathrm{km} / \mathrm{s}$, for 27 stars. Considering that the RV errors are of the order of $10 \mathrm{~km} / \mathrm{s}$ (Fig. 2), we obtain an intrinsic RV dispersion between 21 and $31 \mathrm{~km} / \mathrm{s}$, which is considerably less than that of the Galactic field at similar colors and magnitudes, as indicated by the Besançon model ( $\sim 50 \mathrm{~km} / \mathrm{s}$, Fig. 5, top-middle panel). The RVs of the handful of stars observed at $V \sim 17.5$ and shown in Fig. 2, also indicate a large $(\sim 40 \mathrm{~km} / \mathrm{s})$ RV dispersion, more typical of the Galactic field.

We therefore conclude that the excess blue population between $V=18$ and 19 is a kinematically cold population compared to that of the field, but while Sgr debris is present in SA71, it does not dominate the sample. The other obvious possibility is that this concentration in the VPD is a result of the presence of Monoceros stars. We note that radial-velocity studies within Mon overdensities show RV dispersions that vary between $17 \mathrm{~km} / \mathrm{s}$ and 26 $\mathrm{km} / \mathrm{s}$, with most values around $22 \mathrm{~km} / \mathrm{s}$ (Yanny et al. 2003, Crane et al. 2003, Martin et al. 2006); thus our measurements fit within these determinations. 


\subsubsection{Proper Motions}

We proceed now to investigate the proper-motion distributions of all stars -i.e., not only those with RVs - within the general CMD area where the stellar excess was found (Section 3.1). Since the RVs indicate that the excess stars are NOT predominantly Sgr stars but rather likely Mon members (Section 3.2), we select candidate Mon members from the CMD guided by the two isochrones located at $9 \mathrm{kpc}$ (Fig. 3). First, we select stars within a rectangular area defined between $18 \leq V \leq 21$ and $0.2 \leq B-V \leq 1.2$. There are 428 stars within this sample. Second, we use a more restrictive selection defined along the two isochrones and allowing for small metallicity and distance variations beyond those strictly imposed by the isochrones. This sample is also restricted within the generic magnitude range $V=18$ to 21 , and it includes 213 stars. The CMD selection is illustrated in the top-left panel of Figure 6. The open circles show the box cut, while the filled circles, that along the isochrones. Also, only stars with proper motions based on measurements of six or more plates are considered.

We show the proper-motion diagrams of these Mon-selected samples in Fig. 6. These are absolute proper motions, along Galactic coordinates. The top-right panel shows the box-selected sample; the bottom-left, that of the sample along isochrones. The bottom-right panel of Fig. 6 shows the proper-motion distribution given by the Besançon model within the same CMD box cut applied for the Mon sample selection. The model predicts only 241 stars in the same CMD region where the observations have 428 stars. We have run the Besançon model several times; different realizations provide similar numbers within a $1 \sigma$ uncertainty given by the Poisson statistics. Besides a low-density population with a high proper-motion dispersion, the Besançon Galactic-field distribution shows an elongated shape, oriented at $\sim 45^{\circ}$ with respect to the $\mu_{l} \cos b$ axis. This proper-motion feature can also be distinguished in the observed distributions, in both Mon-selected samples. Along with this feature, there is a significant population clumped around a proper-motion value of $\left(\mu_{l} \cos b, \mu_{b}\right) \sim(0,0)$ mas/yr, that is not present in the model. It is this population that we wish to separate from the Galactic field, and determine its mean absolute proper motion.

We have tried various procedures to separate the field distribution from that of the excess population. First, we have fitted the observed marginal distributions with the sum of two Gaussians, one representing the field, the other the excess population. This is a well-documented technique used when separating clusters from the field in proper-motion space (e.g. Girard et al. 1989). The observed marginal distributions were first constructed along $\left(\mu_{l} \cos b, \mu_{b}\right)$, and then along a $45^{\circ}$-rotated system so that the major axis of the field distribution is along the $x$ proper-motion axis (see Fig. 6). We have worked with both the CMD-box selected sample and the one selected along the isochrones. Second, we have 
done a 2-D subtraction of the field distribution from the observed distribution, by using the Besançon model to represent the field. We have thus constructed density maps in the proper-motion space for the CMD box-selected sample for the observed and the Besançon distributions. We subtract the Besançon density map from the observed one, and determine the centroid of the subtracted density map. Two estimates are provided using this method: one uses the Besançon proper-motion distribution as is, assuming it represents the true Galactic field. The other estimate uses the Besançon proper-motion distribution shifted in proper-motion space so that the elongated feature of the field in the Besançon model is approximately aligned with the same feature in the observed space (see Fig. 6). The adopted shift is determined by eye, and it is $\left(\mu_{l} \cos b, \mu_{b}\right)=(0 ., 1$.$) mas/yr. Internal errors of all$ of these estimates are of the order of a few tenth of mas/yr, and much smaller than the differences between various estimates.

In Table 2 we list the absolute proper motion of the excess population obtained from the various estimates described above. Clearly, the dominant uncertainty in this number is due to the difficulty in separating the field population from the excess population. By using various samples and methods, we aim to quantify this uncertainty. To this end, we

will take the average of the estimates in Tab. 2 as our final determination of the absolute proper motion of the excess population and the scatter ( 1 standard deviation) of the various estimates as the uncertainty of the determination. To this uncertainty we have to add (in quadrature) the error of the proper-motion zero point given by the galaxies (Section 2.1). We thus obtain $\left(\mu_{l} \cos b, \mu_{b}\right)=(0.72 \pm 0.72,0.67 \pm 0.53) \mathrm{mas} / \mathrm{yr}$ for the final absolute proper motion estimate of the blue stellar excess in SA 71.

We have inspected a number of other realizations of the Besançon model that used the same input as the realization discussed here. The VPDs of all realizations are statistically consistent, although the elongated feature discussed above may not distinctly appear in all realizations. This however does not affect our result within its formal error: for instance, the estimation based only on the four measurements that do not include the Besançon model (Tab. 2) give $\left(\mu_{l} \cos b, \mu_{b}\right)=(1.06 \pm 0.64,0.89 \pm 0.43) \mathrm{mas} / \mathrm{yr}$.

\section{Comparison with the Monoceros Tidal Stream Model of Peñarrubia et al.}

We compare our data with the disruption model of P05 that is constrained by the spatial distribution, distance estimates and radial velocities in overdensities mapped above and below the Galactic plane and between $l=110^{\circ}-240^{\circ}$. We note that the model is not constrained by any data in the neighborhood of the CMa overdensity (see Fig. 2 in P05). The model that best explained the data set used by P05 is that of a satellite on a prograde, 
Table 2: Proper-Motion Estimates

\begin{tabular}{lcc}
\hline & & \\
Sample/Method & $\mu_{l} \cos b$ & $\mu_{b}$ \\
\hline CMD box: $0^{\circ}$ & 0.39 & 0.34 \\
CMD box: $45^{\circ}$ & 1.59 & 0.99 \\
& & \\
Isochrone: $0^{\circ}$ & 0.68 & 1.27 \\
Isochrone: $45^{\circ}$ & 1.57 & 0.95 \\
& & \\
2D map - no shift & -0.01 & 0.55 \\
2D map - shift & 0.12 & -0.11 \\
& & \\
Average & 0.72 & 0.67 \\
\hline
\end{tabular}

low-inclination, rather circular orbit.

In Figure 7 we show the spatial distribution in Galactic coordinates of the tidal stream predicted by P05 (grey points). The location of our SA 71 field is indicated with a red symbol. We also show (green line) the orbit of Sgr based on derived proper motions (Dinescu et al. 2005 ) integrated back in time for $\sim 2$ Gyr (some three radial periods). Sgr's orbit precession is from lower $l$ values to higher $l$ values, i.e., moving back in time the orbit is closer to SA 71. From the model, we select a set of points that best represent SA 71's location in the sky; these are shown with black symbols in Fig. 7. Our kinematical results for the candidate Mon population are shown in Figure 8, together with the model predictions. The left panels show the data as a function of $l$, while the right panels show the $b$ dependence. Top and middle panels show the proper motions in Galactic longitude and latitude respectively. The bottom panels show the heliocentric radial velocities. The observed values are shown with a red symbol, and the dark symbols show the selected SA 71-representative sample from the P05 model. For the RV, we indicate the two estimates from Section 3.2.1. The observations are in good overall agreement with the model predictions. The mean radial velocity of the model as given by the representative sample, is different from our determination by $\sim 60 \mathrm{~km} / \mathrm{s}$; however our RV measurement is within the range of predicted values in that region of the sky. Given that the P05 model was constrained rather approximately by the data available then, the agreement with our observations in all kinematic components is remarkable. Using the representative sample from the model, we obtain a RV dispersion of $22 \mathrm{~km} / \mathrm{s}$ as given by probability plots trimmed at $10 \%$ for outliers. This value is compatible with our measurement of the RV dispersion of candidate Mon members (Section 3.2.1). 
Another feature of the model predictions is the shape of the RV distribution. Within a small region of a tidal stream of the model, the RV distributions show sharp edges on one side of the distribution, and this appears to be a generic feature in tidal streams. Our representative sample exemplifies this in Fig. 8 - bottom-right panel. This sharp cutoff is also clearly seen in our RV data (Fig. 5, middle panels, second and fourth rows). Although the sharp edge in the observations is on the opposite side of the velocity distribution than in the model distribution, we are not concerned here with the exact match of the model, but rather with reproducing "generic" features of the model.

Assuming a distance of $9 \mathrm{kpc}$, and adopting the proper motion and radial velocity determined here (the low RV estimate), we obtain an orbit with an eccentricity of $0.09 \pm 0.04$ and an inclination of $20^{\circ} \pm 2^{\circ}$. The orbit integration was done in the potential from Johnston et al. (1995), and the errors are determined from the formal errors in the observables, with an assumed 10\% error in the distance (Dinescu et al. 1999). The higher RV estimate gives an orbit with similar parameters within errors. The orbit determined here is entirely consistent with that from P05.

\section{Other Considerations: The Galactic Warp or More Nearby Overdensities}

The Milky Way warp has been recently re-mapped from the H I distribution by Levine et al. (2006). This work as well as previous others have shown that in the third quadrant, the disk warps below the Galactic plane, with the line of nodes close to the direction to the Anticenter. We also note that the Besançon model includes the Galactic warp and the flare as parametrized with constraints from the near-infrared DENIS survey (Derrière \& Robin 2001).

Nevertheless, we investigate here the possibility that the stellar excess in SA $71((l, b)=$ $\left(167.1^{\circ},-34.7^{\circ}\right)$, second quadrant) may be part of the warp, given that the $\mathrm{H}$ I distribution at longitudes very close to the Anticenter is not well known and that there is an indication that the disk dips under the Galactic plane close to our region of interest (Fig. 6 in Levine et al. 2006). If we assume that the distance to our stellar excess is roughly $9 \mathrm{kpc}$ as indicated by the isochrones (Section 3.1), then this material is at a distance of $\sim 5 \mathrm{kpc}$ below the Galactic plane. As indicated by Levine et al. (2006, their Fig. 6), the displacement of the $\mathrm{H}$ I gas disk below the Galactic plane in the region close to SA 71's longitude is at most $1.3 \mathrm{kpc}$. Therefore the stellar excess in SA 71 can not be interpreted as material from the warp, if the assumed distance is $9 \mathrm{kpc}$. The remaining possibility to investigate is whether the stellar excess is located more nearby than $9 \mathrm{kpc}$. 
If we aim to account for the stellar excess in the brightest magnitude range, namely $V=18$ to 19 (Section 3.1) and that peaks at a color $(B-V) \sim 1.0$, we can envision the main sequence of a nearby stellar overdensity that can pass through this region. For this exercise, we can use our most metal rich isochrone $([\mathrm{Fe} / \mathrm{H}]=-0.7)$ and shift it in magnitude until the main sequence passes through the color range of the stellar excess. This shift amounts to $\sim 3$ mags which corresponds to a population located at $2.3 \mathrm{kpc}$ from the Sun, and $1.3 \mathrm{kpc}$ below the Galactic plane. A more metal rich population - that better represents the disk - would have been shifted less, and therefore the distance to it would have been larger than $2.3 \mathrm{kpc}$. The opposite is true for a more metal poor population than $[\mathrm{Fe} / \mathrm{H}]=$ -0.7. Therefore, we can achieve a reasonable displacement below the Galactic plane of about $1.3 \mathrm{kpc}$ or less, if we assume the overdensity to be relatively metal poor, i.e., $[\mathrm{Fe} / \mathrm{H}] \leq-0.7$, compared to stars in the disk. We note however, that at more nearby distances to the Sun, the displacement of the gaseous disk from the Galactic plane is of the order of a few hundred parsecs (Levine et al. 2006).

The difficulty in considering a more nearby population to explain the stellar excess is not necessarily the metallicity of the population, but the fact that the color of the excess stars is bluer at fainter magnitudes than at brighter magnitudes (Section 3.1, our Fig. 4 and D02). This is opposite from what the main sequence of a nearby population would imply: at fainter magnitudes we should see an excess of stars at redder colors.

For these reasons, we find it unlikely that the stellar excess is part of the Galactic warp, or a stellar overdensity within $2-3 \mathrm{kpc}$ of the Sun.

\section{Conclusions}

Our main findings in area SA 71 that include our previous work (D02) and the current study are the following.

1) We have found a stellar excess of blue stars that is best explained by the existence of an old and rather metal-poor population $(-1.3 \leq[\mathrm{Fe} / \mathrm{H}] \leq-0.7)$ located at $\sim 9 \mathrm{kpc}$. Another possible blue stellar excess can be found in the same region, but at larger distances, i.e., 39 kpc.

2) The proper-motion data for the "bright" ( $V=18-21)$ stellar excess shows a tight clump (smeared only by proper-motion uncertainties) that is indicative of a kinematically cold structure. For the "faint" $(V>21)$ stellar excess, the larger proper-motion errors, as well as the poorer star/galaxy separation do not allow us to draw a definite conclusion.

3) The RVs of the stars in the "bright" excess indicate the existence of a population with a velocity dispersion between 21 and $31 \mathrm{~km} / \mathrm{s}$, which is much lower than that predicted for 
the Galactic field $(50 \mathrm{~km} / \mathrm{s})$. The RV distribution of this population shows a sharp cutoff on one side, unlike the gradual decline of the Galactic field distribution, and its mean RV is between -1 and $10 \mathrm{~km} / \mathrm{s}$. In this stellar excess, we also find ten stars with RVs that indicate their membership to the Sgr tidal stream. The Galactic field should have at most two stars at these extreme RVs $(-180 \mathrm{~km} / \mathrm{s})$. The magnitudes and colors of the Sgr stream candidate members are consistent with a red clump/horizontal-branch population at some $39 \mathrm{kpc}$, thus reinforcing the hypothesis that the "faint" stellar excess is indeed due to turnoff/main sequence stars in the Sgr stream.

4) After separating from the field population, the mean proper motion and RV of the stars in the "bright" excess are compatible with the model predictions for the Monoceros stream by P05. Under the assumption that the debris is located at $9 \mathrm{kpc}$ - another P05 prediction for SA 71 that accounts for the magnitudes and colors of the "bright" excess stars — we determine a prograde orbit of low-inclination and rather circular shape that is consistent with the orbit determined by P05.

5) The sharp edge of the observed RV distribution is similar to that predicted by the P05 model in portions of the stream, and unlike that of the Galactic field. The observed RV dispersion is within the values determined by other studies in different regions of Mon.

6) The uncertainty in the determination of the proper motion and RV for the candidate Mon members is due to the significant overlap with the field population in all three velocity components.

Financial support from NSF grants AST-0406884 and AST-0407207 for this research is acknowledged. J.L.C. is supported by the Virginia Space Grant Consortium.

\section{REFERENCES}

Belokurov, V. et al. 2006, ApJ, 642, L137

Bertin, E., \& Arnouts, S. 1996, A\&AS, 117, 393

Casetti-Dinescu, D. I., Majewski, S. R., Girard, T. M., Carlin, J. L., van Altena, W. F., Patterson, R. J., \& Law, D. R. 2006, AJ132, 2082

Conn, B. C., Lewis, G. F., Irwin, M. J., Ibata, R. A., Ferguson, A. M. N., Tanvir, N., \& Irwin, J. M. 2005, MNRAS, 362, 475

Conn, B. C., Lane, R. R., Lewis, G. F., Gil-Merino, R., Irwin, M. J., Ibata, R. A., Martin, N. F., Bellazzini, M., Sharp, R., Tuntsov, A. V., \& Ferguson, A. M. N., 2007, MNRAS376, 939 
Crane, J. D., Majewski, S. R., Rocha-Pinto, H. J., Frinchaboy, P. M., Skrutskie, M. F., \& Law, D. R. 2003, ApJ594, L119

Derrière, S. \& Robin, A. C. 2001, in ASP Conf. Ser. Vol. 232, "The New Era of Wide Field Astronomy" ed. R. Clowes, A. Adamson and G. Bromage

Dinescu, D. I., Girard T. M., \& van Altena, 1999, AJ117, 1792

Dinescu, D. I., Majewski, S. R., Girard, T. M., Méndez, R. A., Sandage, A., Siegel, M. H., Kunkel, W. E., Subasavage, J. P., \& Ostheimer, J. 2002, ApJ, 575, 70

Girard, T. M., Grundy, W. M., López, C. E., \& van Altena, W. F. 1989, AJ98, 227

Girardi, L., Bertelli, G., Bressan, A., Chiosi, C., Groenewegen, M. A. T., Marigo, P., Salasnich, B., \& Weiss, A. 2002, A\&A391, 195

Grillmair, C. 2006, ApJ651, L29

Hamaker, H. C. 1978, Applied Statistics, 27, 76

Ibata, R. A., Irwin. M. J., Lewis, G. F., Ferguson, A. M. N., \& Tanvir, N. 2003, MNRAS, 340,21

Johnston, K. V., Spergel, D. N., \& Hernquist, L. 1995, ApJ, 451, 598

Law, D. R., Johnston, K. V., \& Majewski, S. R. 2005, ApJ619, 807

Levine, E. S., Blitz, L., \& Heiles, C. 2006, ApJ643, 881

Majewski, S. R., Skrutskie, M. F., Weinberg, M. D., \& Ostheimer, J. C. 2003, ApJ, 599, 1115

Majewski, S. R., et al. 2004, AJ128, 245

Martin, N. F., Irwin, M. J., Ibata, R. A., Conn, B. C., Lewis, G. F., Bellazzini, M., Chapman, S., \& Tanvir, N. 2006, MNRAS367, L69

Mathieu, R. D., Latham, D. W., Griffin, R. F., \& Gunn, J. E., 1986, AJ92, 1100

Méndez, R. A., \& van Altena, W. F. 1996, AJ112, 655

Newberg, H. J. et al. 2002, ApJ, 569, 245

Peñarrubia, J., Martínez-Delgado, D., Rix, H. W., Gómez-Flechoso, M. A. Munn, J., Newberg, H., Bell. E. F., Yanny, B., Zucker, D. \& Grebel, E. K. 2005, ApJ, 626, 128 (P05)

Putman, M. E., Thom, C., Gibson, B. K., Staveley-Smith, L. 2004, ApJ603, L77

Robin, A. C., Reylé, C., Derrière, \& Picaud, S. 2003, A\&A, 409, 523

Rocha-Pinto, H. J., Majewski, S. R., Skrutskie, M. F. \& Crane, J. D. 2003, ApJ594, 115 
Rocha-Pinto, H. J., Majewski, S. R., Skrutskie, M. F., Crane, J. D., \& Patterson, R. J. 2004, ApJ, 615, 732

Schlegel, D. J., Finkbeiner, D. P., \& Davis, M. 1998, ApJ, 500, 525

Tonry, J., \& Davis, M. 1979, AJ84, 1511

Totten, E. J., \& Irwin, M. J. 1998, MNRAS294, 1

Vivas, A. K., \& Zinn, R. 2006, AJ132, 714

Yanny, B., Newberg, H. J., Grebel, E. K., Kent, S., Odenkirchen, M., Rockosi, C. M.,

Schlegel, D., Subbarao, M., Brinkmann, J., Fukugita, M., Ivezik, Z., Lamb, D. Q., Schneider, D. P., \& York, D. G. 2003, ApJ, 588, 824 

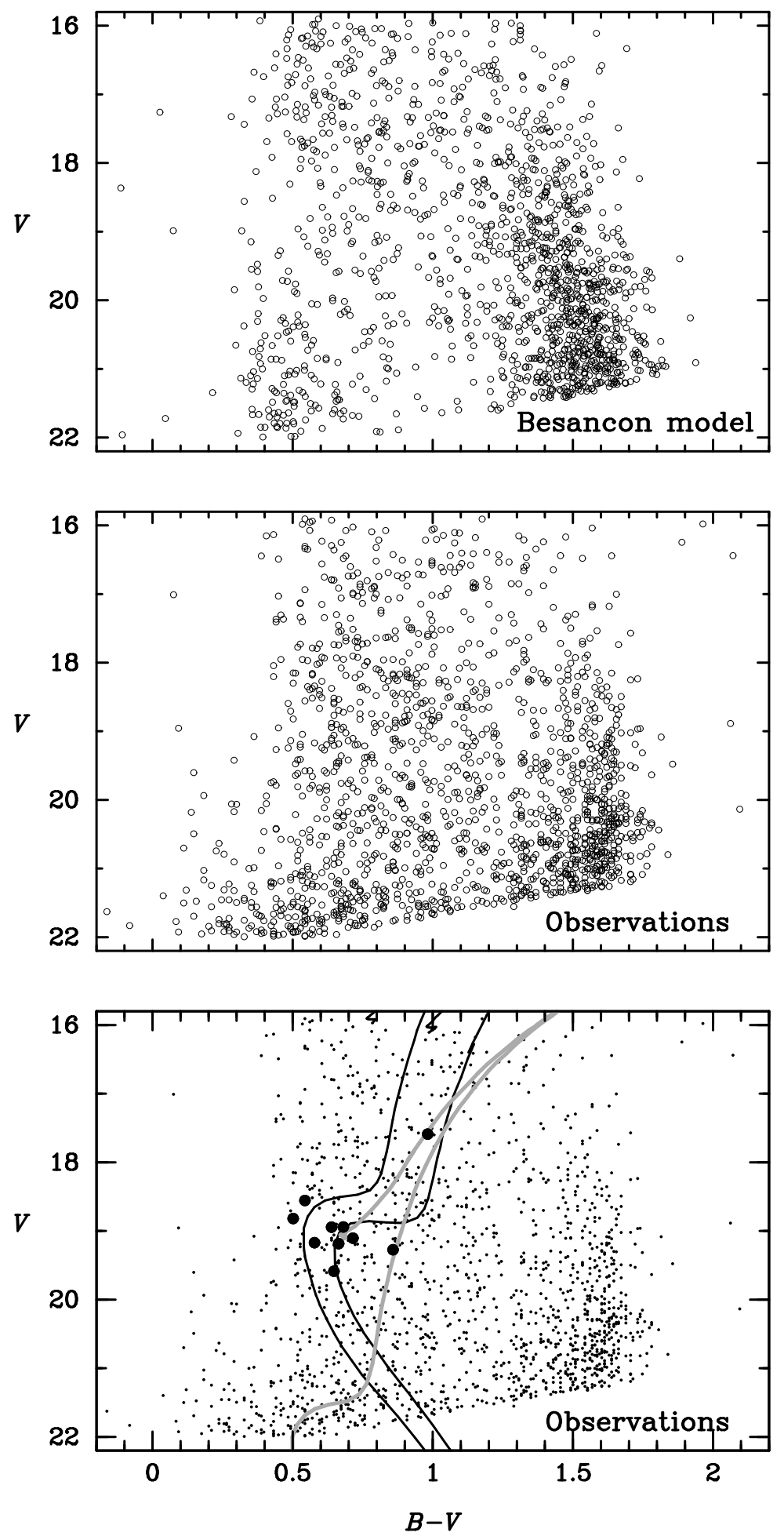

Fig. 3.- CMDs for the Besançon model (top) and for our observations (middle and bottom panels). In addition to the observations, the bottom panel includes two 10 Gyr isochrones at $d_{\odot}=9 \mathrm{kpc}$ and $[\mathrm{Fe} / \mathrm{H}]=-1.3$ and -0.7 representing Mon, and one at $d_{\odot}=39 \mathrm{kpc}$ and $[\mathrm{Fe} / \mathrm{H}]=-1.7$ representing Sgr. The dark symbols in the bottom panel show the Sgr candidate members according to RVs. 

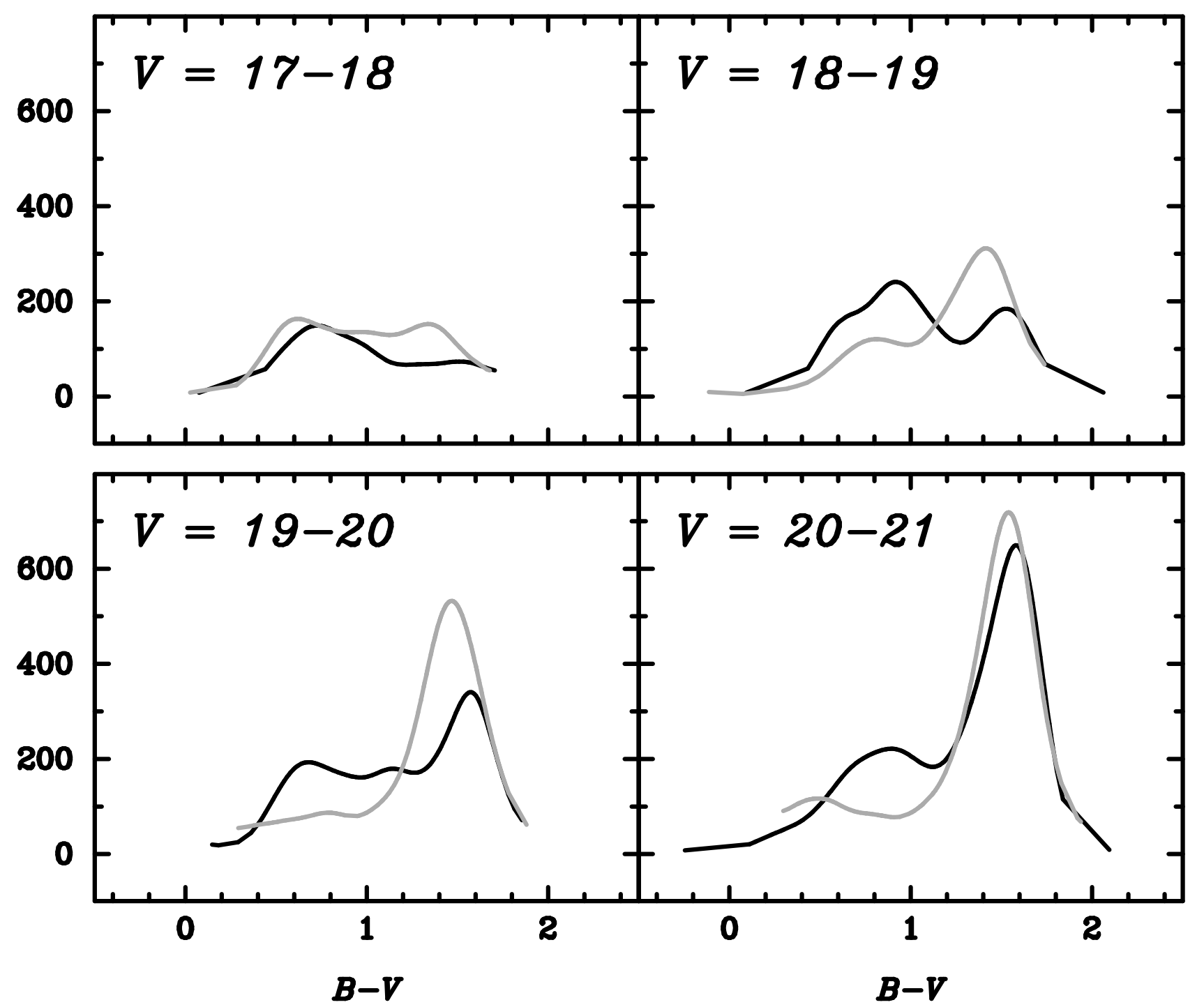

Fig. 4.- Color distributions of the observations (black line) and of the Besançon model predictions (grey line) for four magnitude bins, as specified in each panel. 

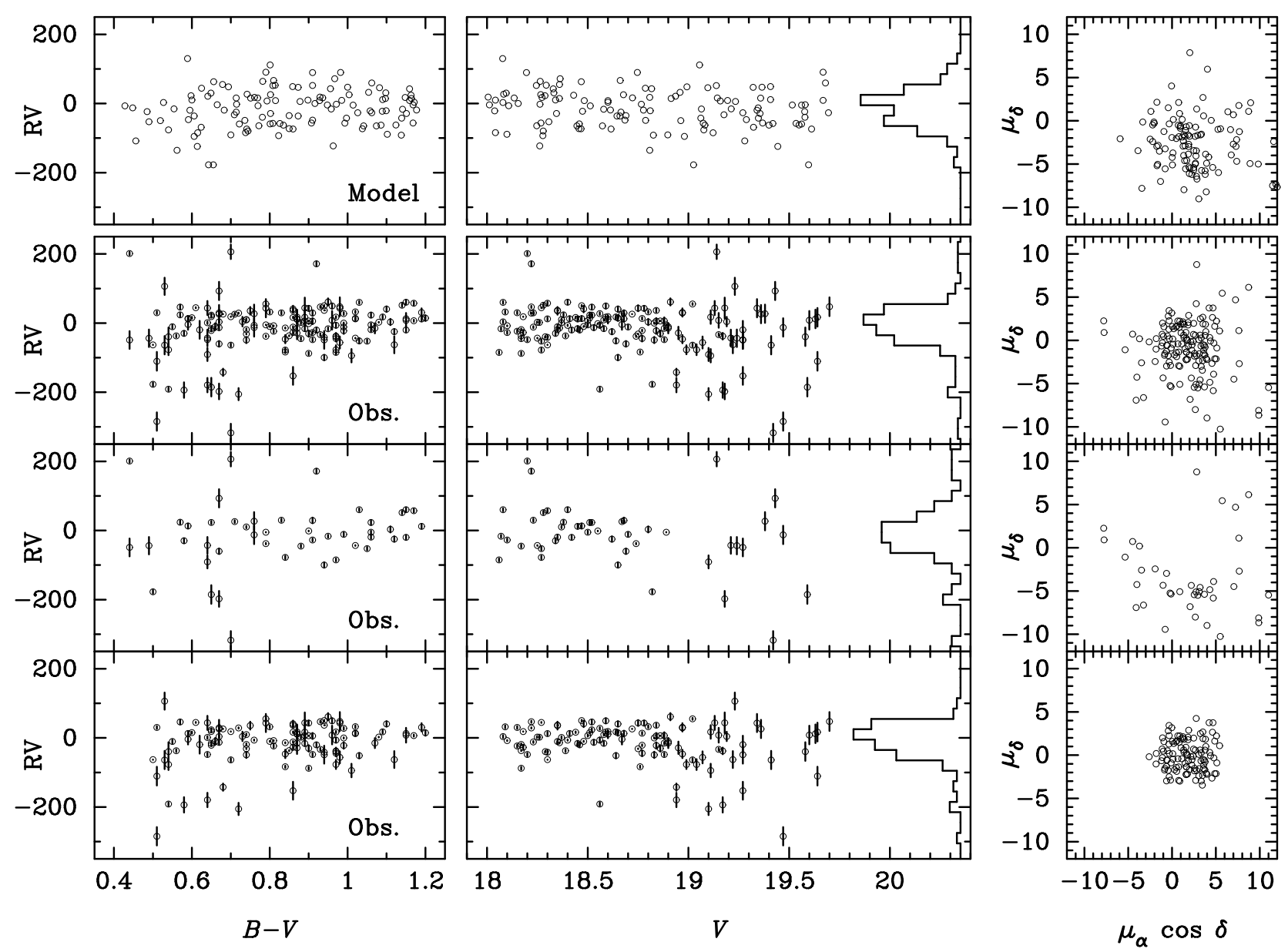

Fig. 5.- RVs as a function of color (left) and magnitude (middle) for the Besançon model (top row) and for various samples of our observations (second to fourth rows). The right panels show the proper-motion distributions of the respective samples. The second row shows all of the RV observations, the third, those outside the proper-motion clump, and the fourth, those inside the proper-motion clump. 


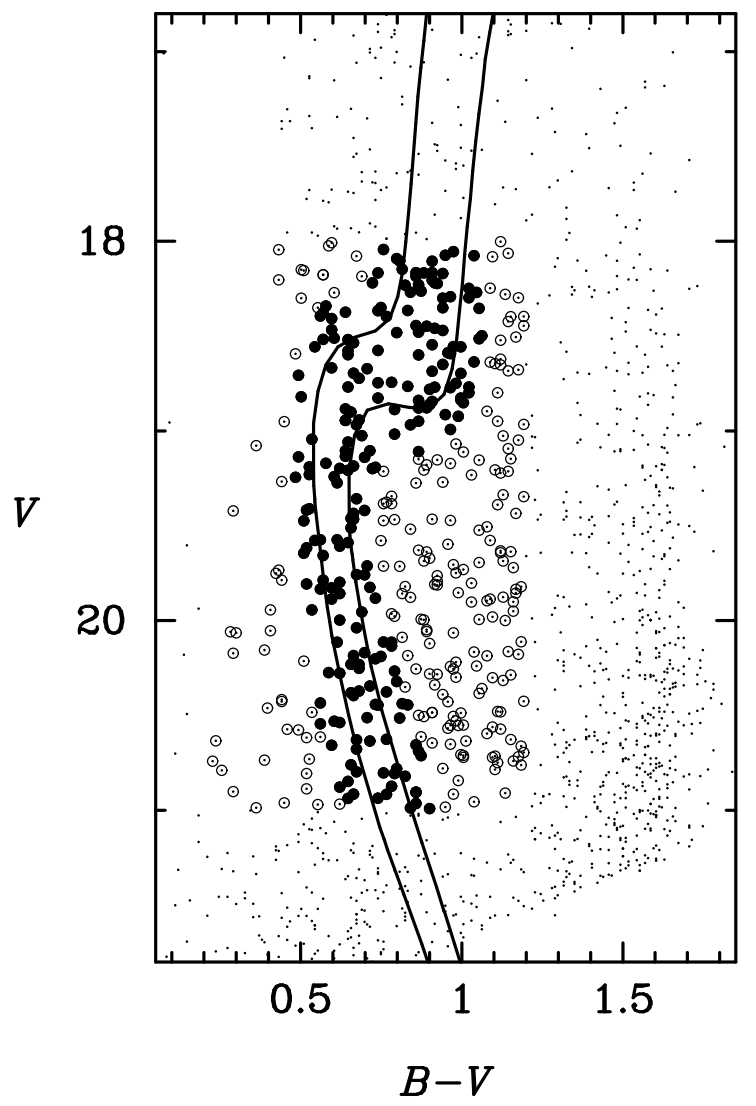

Obs. - Along isochrones

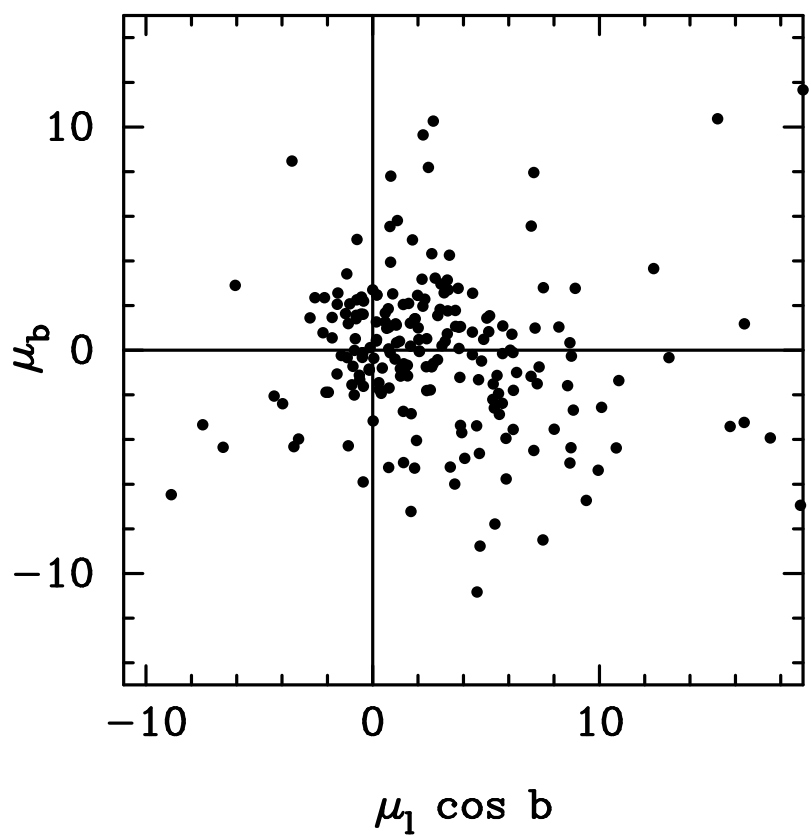

Obs. - CMD box

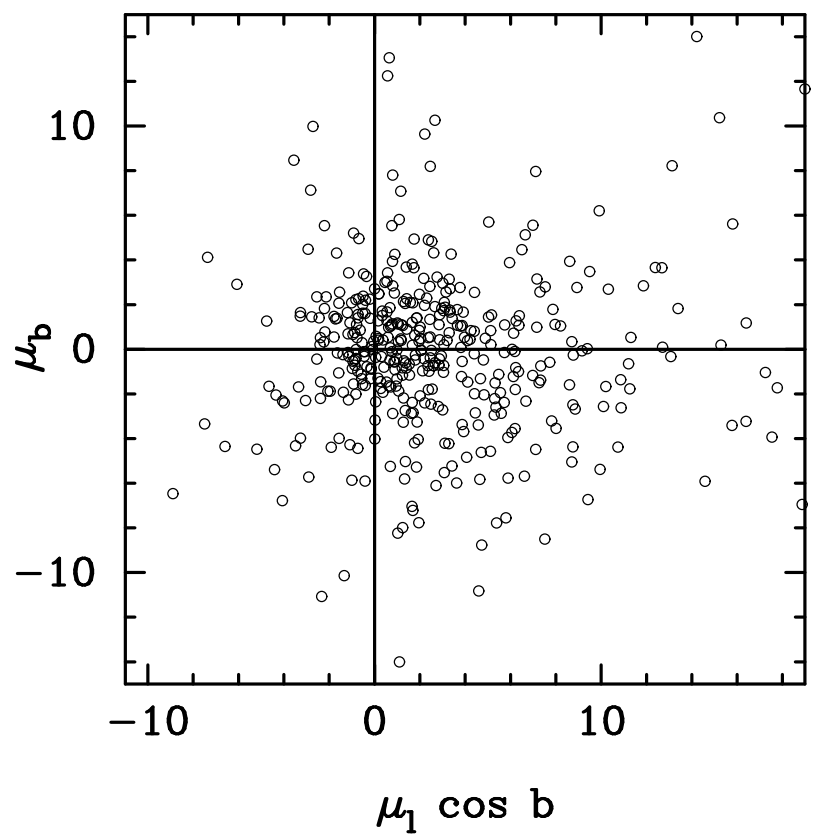

Besancon - CMD box

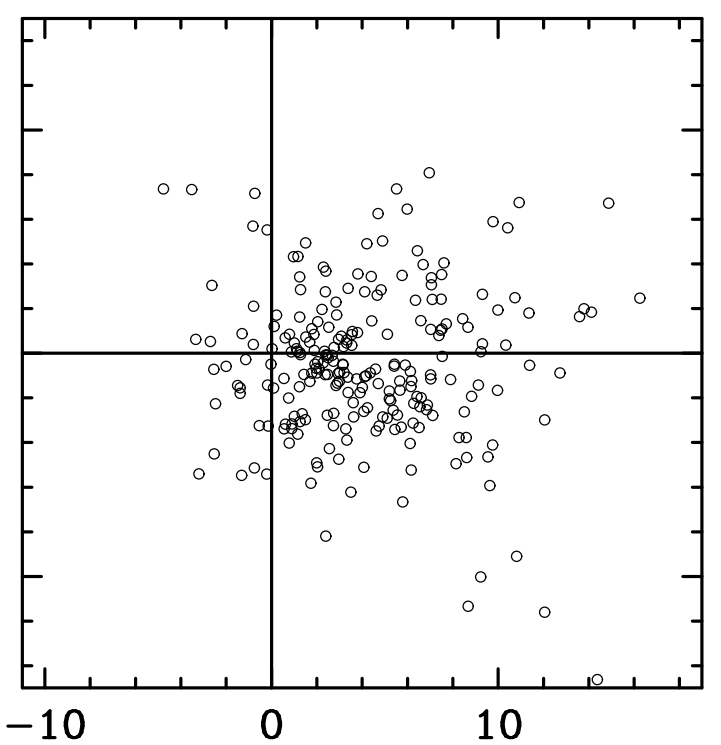

$\mu_{1} \cos \mathrm{b}$

Fig. 6.- The CMD selection (top-left) of candidate Mon members: a box selection (open circles) and an isochrone-guided selection (filled circles). The proper-motion distributions of the CMD-box selected members (top-right) and isochrone-selected members (bottom-left). The Besançon model distribution for the same CMD-box selection is show in the bottom- 


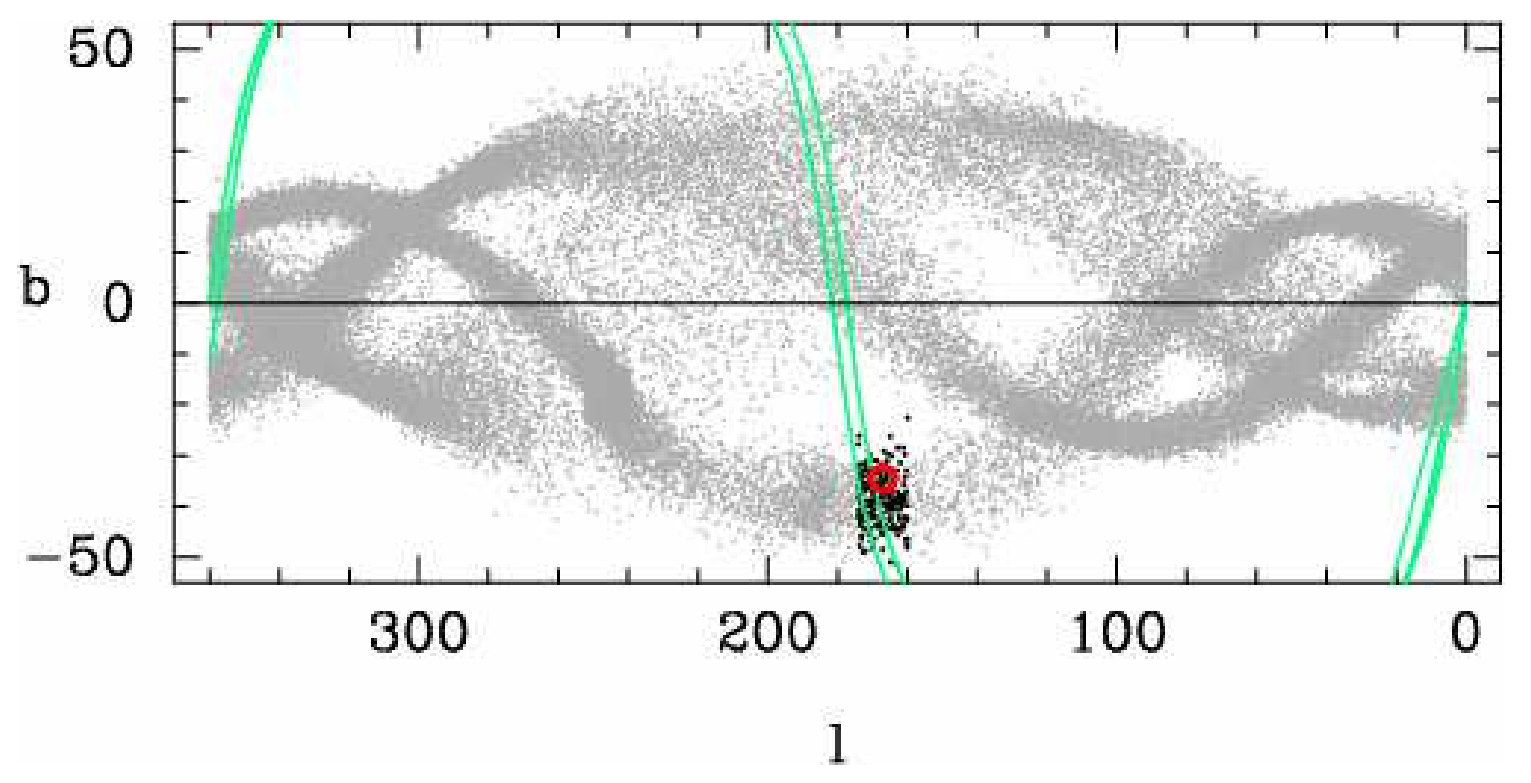

Fig. 7.- The spatial distribution of Mon tidal debris from the model of P05 (grey points). In green we show the orbit of Sgr, and in red the location of SA 71. The dark points show the SA71-representative sample selected from the P05 model. 

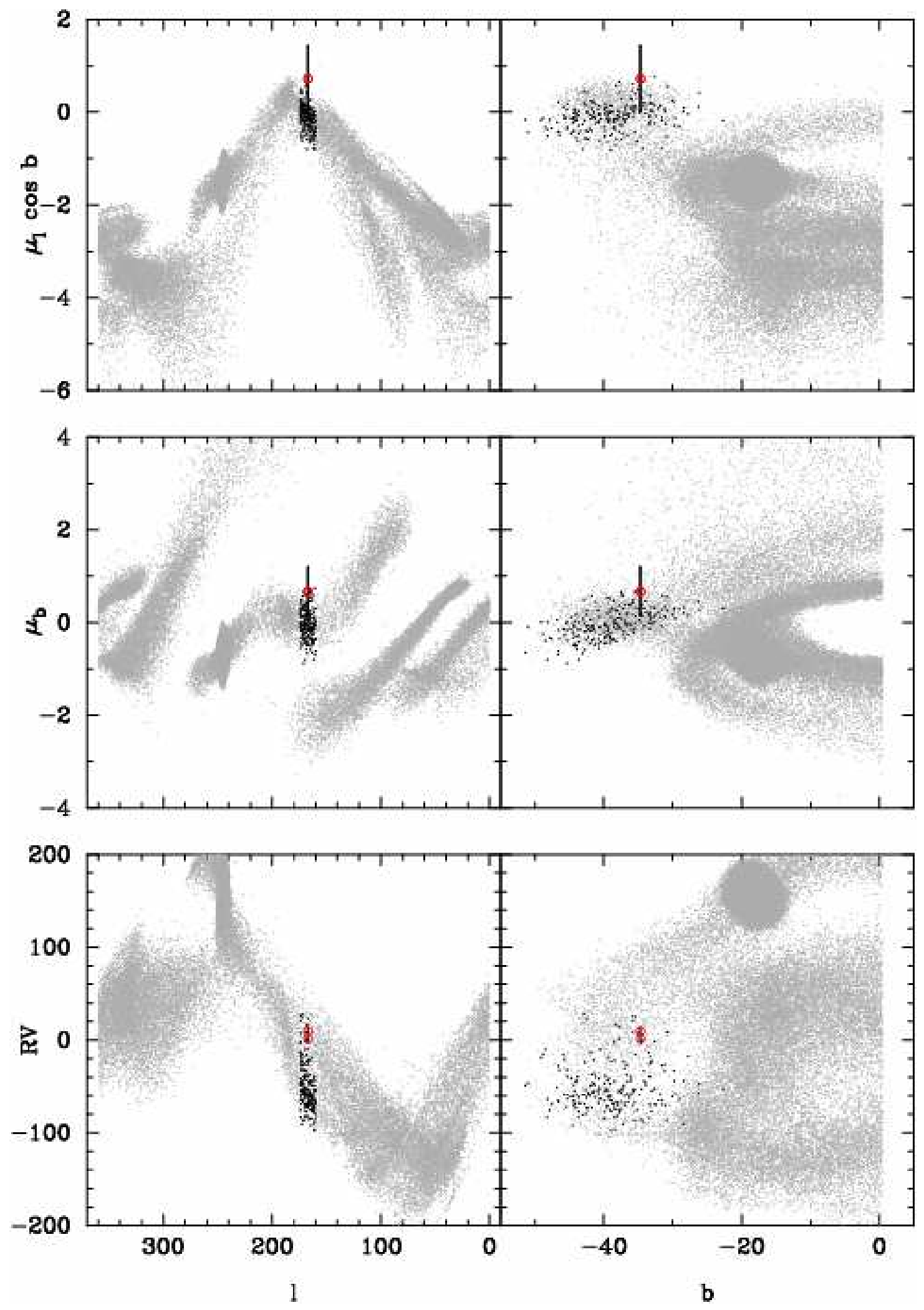

Fig. 8. - Comparison of the P05 kinematical predictions with the observations in SA 71. The left panels show the dependence with Galactic longitude, while the right panels that with Galactic latitude; we restrict the comparison to values of $b \leq 0^{\circ}$. The grey points show the model predictions and the dark points those of the SA71-representative sample. In red 